Trauma Berufskrankh 2010 - 12[Suppl 2]:151-152 DOI 10.1007/s10039-010-1625-8

Online publiziert: 8. Mai 2010

(c) Springer-Verlag 2010

\title{
M. Folk
}

Orthopädisch-chirurgisches Versorgungszentrum Markt Schwaben

\section{Praxis des ambulanten Durchgangsarztverfahrens}

der Unfallverletzten neue Probleme. Der neue Facharzt für Allgemeinmedizin wird in Zukunft ohne praktische Tätigkeit in der Chirurgie erworben und für die Erst- oder Weiterbehandlung von Unfallverletzten keine medizinische Kompetenz mehr besitzen. Auch der künftige Facharzt für Allgemein und Viszeralchirurgie wird in Zukunft keine traumatologischen Kenntnisse mehr besitzen. Beide derzeit noch sehr intensiv in die Behandlung dieser Klientel eingebundenen Facharztsparten scheiden somit zukünftig für die Versorgung der Unfallverletzten im berufsgenossenschaftlichen Bereich aus.

Durch einen zunehmenden Nachwuchsmangel im Bereich der operativen Fächer wird sich die Versorgungslage noch zunehmend verschärfen.

Im Zuge der Spezialisierung der Abteilungen im Krankenhaussektor wird es zu einer abnehmenden Erfahrung der zukünftigen Durchgangsärzte im Bereich der ambulanten Erst- und Weiterbehandlung kommen. Durch die Beschränkung der Tätigkeit im Spezialgebiet können auch keine Erfahrungen mehr in den angrenzenden Fachgebieten wie Gefäßchirurgie, Viszeralchirurgie, Handchirurgie oder Anästhesie gesammelt werden.

\section{Derzeitige Schwachstellen des D-Arzt-Verfahrens}

Aktuelle Probleme, die in Klinik und Praxis bei der Erstbehandlung Unfallverletzter auftreten, sollten bei der Weiterentwicklung des D-Arzt-Verfahrens berücksichtigt werden. Im Bereich der selbstständig ambulant tätigen Durchgangsärz- te sind diesbezüglich v. a. folgende Probleme von Bedeutung:

- Die Integration der Versorgung von Verletzten in einen geregelten Ablauf einer Bestellpraxis mit entsprechenden Terminverschiebungen gelingt nur schwer.

- Die Verfügbarkeit von Ressourcen wie Anästhesie, bildgebende Diagnostik oder Laboruntersuchungen ist oft eingeschränkt.

- Die Unfallbereitschaft des Durchgangsarztes kann zu den geforderten Zeiten, z. B. am Samstag, nicht adäquat aufrechterhalten werden.

Im stationären Sektor stehen andere Problematiken im Vordergrund:

- Aufgrund der Dienstverteilung gelingt es kaum einer Klinik, jeden Verletzten durch einen Facharzt für Unfallchirurgie behandeln zu lassen.

- Durch die von einigen Kliniken propagierte „Hausarztpflege“ erfolgt die weiterführende Behandlung oft durch einen möglicherweise nicht ausreichend qualifizierten Arzt.

- Häufig ist auch die Befundweitergabe problematisch: D-Arzt-Berichte und Röntgenbilder werden nicht oder nur verzögert weitergegeben. Die CD mit den Röntgenbildern enthält oft überflüssige „Werbesoftware“ des Herstellers, was den Zugriff auf wichtige Daten erheblich verzögert. Hier sind in Zukunft detaillierte Anforderungen auch von berufsgenossenschaftlicher Seite zur reibungslosen Befundweitergabe erforderlich.
Durch die Reformierung der Weiterbildung ergeben sich in der Versorgung 
Trauma Berufskrankh 2010 - 12[Suppl 2]:

151-152

DOI 10.1007/s10039-010-1625-8

c) Springer-Verlag 2010

\section{Folk \\ Praxis des ambulanten Durchgangsarztverfahrens}

\section{Zusammenfassung}

Die Rahmenbedingungen für das Durchgangsarztverfahren haben sich in den letzten Jahren erheblich gewandelt, weshalb eine Neuordnung und Weiterentwicklung dringend geboten erscheint. Diese sollte die geänderten Fachgebietsbezeichnungen und die Ausbildung der Durchgangsärzte, die durch die Reformierung der Weiterbildung und die damit einhergehende Spezialisierung zu befürchtenden Engpässe in der Versorgung der Unfallverletzten und die aktuellen Problematiken der niedergelassenen und stationär tätigen Durchgangsärzte berücksichtigen. Hierzu ist dringend eine Einbeziehung der ambulant tätigen Durchgangsärzte erforderlich, da deren Belange durch die kassenärztliche Bundesvereinigung nur ungenügend vertreten werden.

\section{Schlüsselwörter}

Durchgangsarztverfahren · Fachgebietsbezeichnung · Ausbildung · Weiterbildung . Ambulant tätiger Durchgangsarzt

\section{Out-patient authorized medical examinations in practice}

\begin{abstract}
The basic parameters for authorized medical examination procedures have changed considerably in recent years, appearing to make reorganization and enhancement urgently necessary. The factors to be taken into consideration include altered specialty designations and authorized medical examiner training, which, as a result of reforms to further medical training and associated specialization, may lead to bottlenecks in the treatment of accident injuries, as well as the current problems accompanying authorized medical examiners active either in practices or in-patient departments. Inclusion of the authorized medical examiner active in outpatient departments in any reorganization is urgently required, since his interests are currently under-represented by the German national association of statutory health insurance physicians.
\end{abstract}

\section{Keywords}

Authorized medical examiner - Specialty designation - Training · Further training . Out-patient medical examiner

\section{Weiterentwicklung des D-Arzt-Verfahrens im ambulanten Bereich}

\section{Problematiken}

Bei der diesbezüglichen Diskussion haben die niedergelassenen Ärzte nach Meinung des Autors ein zu geringes Mitspracherecht. Die Vertretung durch die kassenärztliche Bundesvereinigung ist ungenügend, da ein direkter Bezug des verhandelnden Gremiums zur Basis der Durchgangsärzte fehlt.

Bei den örtlichen kassenärztlichen Vereinigungen sind bei der Umsetzung der D-Arzt-Nachfolge große Unsicherheiten vorhanden; so ist in Bayern die Nachfolge für einen D-ärztlichen Chirurgen ohne Schwerpunktbezeichnung „Unfallchirurgie“ durch einen „Facharzt für Unfallchirurgie und Orthopädie“ nicht möglich (Aussage Zulassungsausschuss April 2009). Ebenso ungeklärt ist die veränderte Bedarfsplanung durch den Zusammenschluss der Fächer Unfallchirurgie und Orthopädie.

Durch die starke Abhängigkeit des ambulanten Sektors vom Gesetzgeber wurde in den letzten Jahren eine massive Unsicherheit erzeugt. Während durch teilweise unsinnige Gesetzesvorgaben die Praxiskosten und der Verwaltungsaufwand massiv erhöht wurden, ist die Honorierung durch die Gesetzliche Krankenversicherung unsicherer den je. So muss nach Vorgaben des SGB (Sozialgesetzbuch) V die Behandlung ,wirtschaftlich und ausreichend" sein, weswegen ein hoher Behandlungsaufwand nicht mehr in der Gebührenordnung abgebildet wird. Demgegenüber steht das berufsgenossenschaftliche Verfahren, bei dem die rasche Wiederherstellung der Arbeitsfähigkeit des Unfallverletzten möglichst ohne Einschränkungen im Vordergrund steht.

\section{Ziele}

Aus der Sicht der niedergelassenen DÄrzte sind mehrere Faktoren wichtig:

- Die Qualifikation des D-Arztes ist mit der Anerkennung „Facharzt für Orthopädie und Unfallchirurgie" sowie einer 2 jährigen Tätigkeit als Facharzt an einem Haus mit Anerkennung zum Verletztenartenverfahren (VAV) ausreichend. Die Zulassung muss aber auch für Eingriffe des stationsersetzenden ambulanten Operierens geltend.

- Die Zulassung zum D-Arzt muss dessen Kollegen in Berufsausübungsgemeinschaften in die Lage versetzen, im Rahmen deren Fachgebietes bei der Behandlung von Unfallverletzten tätig zu werden.

- Die Unfallbereitschaft der ambulanten D-Ärzte muss an die Präsenzpflicht im kassenärztlichen Bereich angepasst werden, diese kann z. B. an Samstagen im Niedergelassenenbereich nicht mehr erbracht werden.

- Die Lotsenfunktion des niedergelassenen D-Arztes muss ausgebaut werden, insbesondere sollte die derzeitige Behandlung der allgemeinen Heilbehandlung durch nicht ausreichend in der Unfallbehandlung ausgebildete Ärzte unterbleiben. Eine Arbeitsunfähigkeitsbescheinigung dürfte dann nur noch vom D- oder H-Arzt (Heilbehandlungsarzt) ausgestellt werden.

- Bei der Weiterentwicklung der BG GOÄ (Gebührenordnung für Ärzte für die Leistungs- und Kostenabrechnung mit den Berufsgenossenschaften) sind betriebswirtschaftliche Erfordernisse ausgeprägter als bisher zu berücksichtigen, hier sollten in Zukunft die entsprechenden Berufsverbände eingebunden werden. Insbesondere im Bereich des ambulanten Operierens sind derzeit teilweise groteske Missverhältnisse vorhanden. Auch bei der Weiterentwicklung der „Arbeitshinweise zur Prüfung von Arztrechnungen“ ist die Beratung durch die Fachgesellschaften erforderlich, da medizinische und juristische Erfordernisse teilweise nur unzureichend berücksichtigt werden.

\section{Korrespondenzadresse}

Dr. M. Folk

Orthopädisch-chirurgisches Versorgungszentrum Markt Schwaben,

Bahnhofstraße 25, 85570 Markt Schwaben praxis@orthochirurg.de

Interessenkonflikt. Der korrespondierende Autor gibt an, dass kein Interessenkonflikt besteht. 